\title{
Co-enrollment in multiple HIV prevention trials - Experiences from the CAPRISA 004 Tenofovir gel trial
}

\author{
Quarraisha Abdool Karim a,b, Ayesha B.M. Kharsany ${ }^{\mathrm{a}, *}$, Kasavan Naidoo ${ }^{\mathrm{a}}$, Nonhlanhla Yende ${ }^{\mathrm{a}}$, \\ Tanuja Gengiah ${ }^{a}$, Zaheen Omar ${ }^{a}$, Natasha Arulappan ${ }^{a}$, Koleka P. Mlisana ${ }^{a}$, \\ Londiwe R. Luthuli ${ }^{a}$, Salim S. Abdool Karim ${ }^{\text {a,b,c }}$ \\ a Centre for the AIDS Program of Research in South Africa (CAPRISA), Durban, South Africa \\ b Department of Epidemiology, Mailman School of Public Health, Columbia University, NY, USA \\ c University of KwaZulu-Natal, Durban, South Africa
}

\section{A R T I C L E I N F O}

\section{Article history:}

Received 26 October 2010

Accepted 12 January 2011

\section{Keywords:}

HIV prevention trials

Co-enrollment

Young women

\begin{abstract}
A B S T R A C T
Background: In settings where multiple HIV prevention trials are conducted in close proximity, trial participants may attempt to enroll in more than one trial simultaneously. Co-enrollment impacts on participant's safety and validity of trial results. We describe our experience, remedial action taken, inter-organizational collaboration and lessons learnt following the identification of co-enrolled participants.

Experiences: Between February and April 2008, we identified 185 of the 398 enrolled participants as ineligible. In violation of the study protocol exclusion criteria, there was simultaneous enrollment in another HIV prevention trial (ineligible co-enrolled, $\mathrm{n}=135$ ), and enrollment of women who had participated in a microbicide trial within the past 12 months (ineligible not coenrolled, $n=50$ ). Following a complete audit of all enrolled participants, ineligible participants were discontinued via study exit visits from trial follow-up. Custom-designed education program on co-enrollment impacting on participants' safety and validity of the trial results was implemented. Shared electronic database between research units was established to enable verification of each volunteer's trial participation and to prevent future co-enrollments.

Lessons learnt: Interviews with ineligible enrolled women revealed that high-quality care, financial incentives, altruistic motives, preference for sex with gel, wanting to increase their likelihood of receiving active gel, perceived low risk of discovery and peer pressure are the reasons for their enrollment in the CAPRISA 004 trial.

Conclusion: Instituting education programs based on the reasons reported by women for seeking enrollment in more than one trial and using a shared central database system to identify coenrollments have effectively prevented further co-enrollments.
\end{abstract}

c) 2011 Elsevier Inc. All rights reserved.

\section{Introduction}

Sub-Saharan Africa continues to experience high HIV incidence rates [1], and is therefore an important region for

\footnotetext{
* Corresponding author at: Centre for the AIDS Program of Research in South Africa, 2nd Floor, Doris Duke Medical Research Institute, Nelson R Mandela School of Medicine, University of KwaZulu-Natal, Private Bag X7, Congella 4013, South Africa. Tel.: +27 31 2604558; fax: +27 312604566.

E-mail address: kharsany@ukzn.ac.za (A.B.M. Kharsany).
}

carrying out HIV prevention trials in order to test potential biomedical interventions to curb the current pandemic [2]. A systematic review of 37 HIV prevention randomized control trials (RCTs) showed that approximately 90\% demonstrated no significant effects on HIV incidence [3]. Reasons for these results could be a failure in the underlying conceptualization of the intervention, an ineffective intervention, provision of enhanced prevention packages in the comparison arm or failure to monitor and correct shortcomings in trial implementation $[2,3]$. 
Nevertheless, RCTs are the gold standard for evaluating the effectiveness of an intervention [3] and provide data on the appropriateness and feasibility of the intervention, upon which clinical practices can be based. The strict eligibility criteria may limit the external validity and generalizability of the findings, yet the scientific integrity of the RCT in its inherent design minimizes the effects of confounding variables with each participant having the same chance of receiving a test intervention and both measured and unmeasured confounding variables are considered to be equally distributed amongst the study arms [4].

Almost 15,000 women in sub-Saharan Africa have participated in HIV prevention trials. The trial sites are usually geographically isolated, and participants are recruited from separate communities or catchment areas [5-10]. However, with increasing numbers of interventions tested in parallel such as microbicides, vaccines, cervical barriers, and structural changes, geographic isolation of trial sites is no longer guaranteed, making it possible for trial participants to participate in more than one trial simultaneously. There are many reports on factors that motivate volunteers to participate in clinical trials [11-13] but little attention has been paid to the reasons for co-enrollment or participation in multiple trials simultaneously [11,14]. Key elements of research participation are governed by human subject research protection of respect, beneficence, and justice and to preserve scientific integrity and to maintain the safety of trial participants.

This paper describes the identification of the co-enrollment of participants in the CAPRISA 004 Tenofovir gel and other HIV prevention trials, steps taken to rectify the problem, implementation of inter-organizational collaboration to prevent recurrences and lessons learnt.

\section{Experiences}

\subsection{CAPRISA 004 Tenofovir gel trial}

CAPRISA 004 was a Phase IIb double-blind RCT designed to establish the safety and effectiveness of $1 \%$ Tenofovir gel in preventing HIV infection in women in South Africa (CAPRISA 004 trial) [9]. The trial was conducted at two CAPRISA clinics; the urban CAPRISA eThekwini Clinic in the central business district of Durban and the rural Vulindlela Clinic, $150 \mathrm{~km}$ inland from Durban. The trial was initiated in May 2007 with an enrollment target of 920 participants. During cohort accrual for CAPRISA 004 at the urban and rural clinics, another research organization had several active HIV prevention trials underway in the greater Durban area (Fig. 1), at sites a substantial distance away from the CAPRISA urban clinic.

Recruitment strategies at the urban clinic initially targeted women attending the adjacent local sexually transmitted disease (STD) clinic; self-identified sex workers from surrounding areas and women transitioning from two other CAPRISA cohort studies [15,16]. However, enrollment was also open to women from the general population.

During the planning of the CAPRISA 004 trial, the potential for co-enrollment was considered as a cellulose sulphate microbicide trial site was within $5 \mathrm{~km}$ of the urban site (Fig. 1). Initial concern about co-enrollment dissipated when the cellulose sulphate trial closed several months prematurely before the CAPRISA 004 trial commenced.

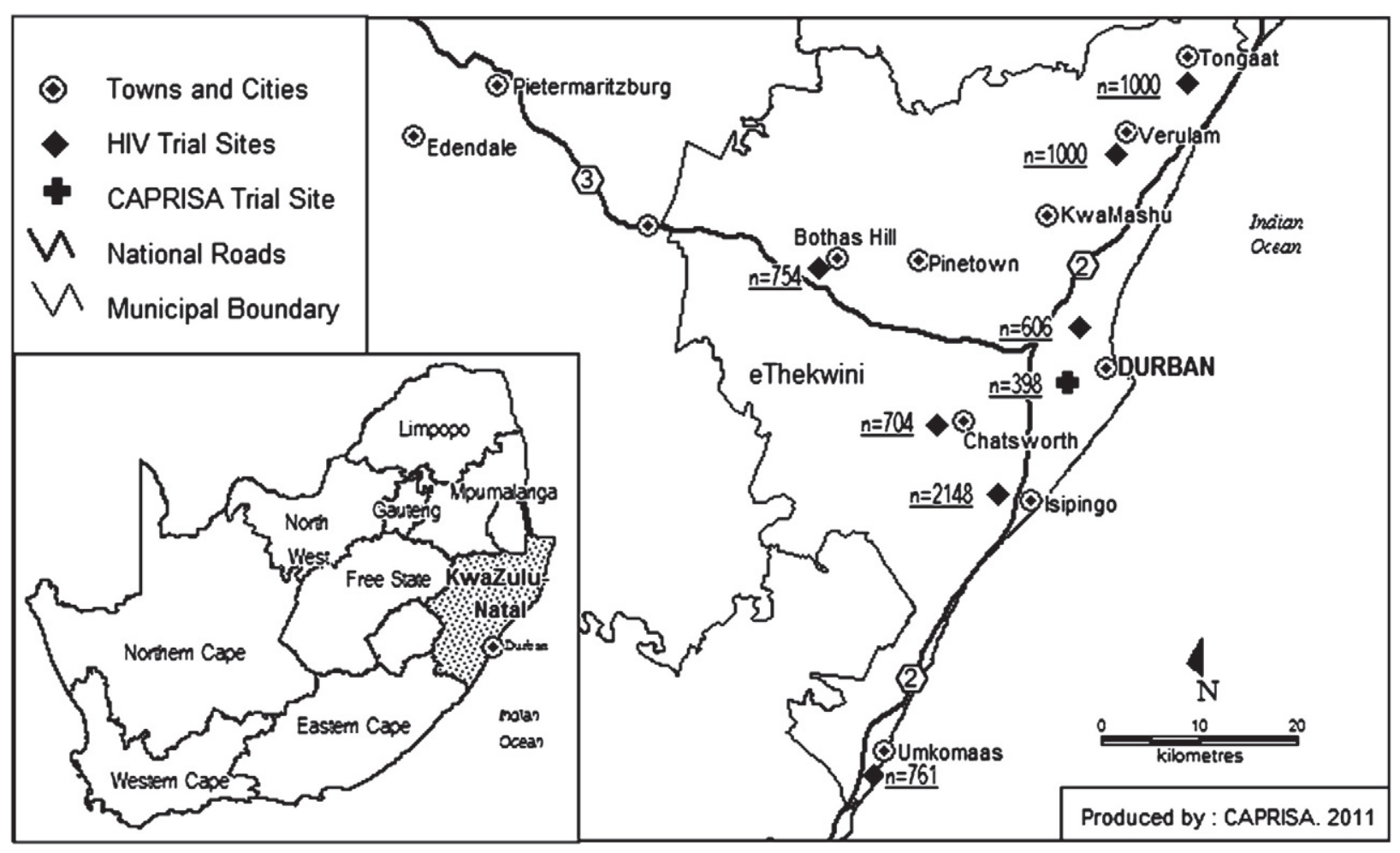

Fig. 1. HIV prevention trial sites in the greater Durban area, KwaZulu-Natal, South Africa. 
Even though a key exclusion criterion included self-report of current enrollment in any other HIV prevention trial, or participation in such a trial within the past 12 months, we made three assumptions about co-enrollment:

- Following the premature closure of the cellulose-sulphate trial, the distance between the urban site and the closest other microbicide trial site was $25 \mathrm{~km}$ away. We assumed that participants would not travel this distance to participate simultaneously in another clinical trial.

- The primary recruitment was done through the STD clinic which was not a recruitment site for other organizations conducting HIV prevention trials. We assumed that there would be no overlap with recruitment.

- The trial site staff were confident that self-report of current or recent non-participation in another trial of vaginally applied product or behavioural study would be reliable. We assumed that participants would be honest in their responses.

This false sense of security meant that there were no additional systems in place to verify self-reports and identify co-enrolled participants.

\subsection{Discovery of co-enrolled trial participants}

Two distinct incidents led to the discovery of coenrollment. Early in February 2008, a research nurse reviewing participant family planning cards identified two participants who disclosed that they were currently participating in another microbicide trial. At about the same time during a study monitoring visit, a monitor identified amongst returned study applicators a study applicator from another microbicide trial. Pharmacy staff who subsequently instituted more rigorous review of used applicators found another participant returning applicators from another trial.

A full audit was launched at both CAPRISA sites and the extent of co-enrollment was established by April 2008 as confined to the CAPRISA urban clinic. Three categories of trial participants were identified:

- Ineligible co-enrolled: participants who were participating in two HIV prevention trials simultaneously. These participants were participating in a trial of a vaginally applied product or behaviour modification for HIV prevention first and then enrolled in CAPRISA 004 trial.

- Ineligible not co-enrolled: participants enrolled in CAPRISA 004 trial only, but who had participated in a trial of a vaginally applied product within the last 12 months.

- Eligible enrolled: participants who met all the eligibility criteria for enrollment into CAPRISA 004 trial.

At the CAPRISA urban clinic, 185 of the 398 enrolled participants were either ineligible co-enrolled $(n=135)$ or were ineligible not co-enrolled $(n=50)$. As these participants did not meet the pre-randomization criteria, they were terminated from CAPRISA 004.

A further 4 participants were found to have enrolled in another microbicide trial after they had enrolled in CAPRISA 004 trial. Since these participants met the eligibility criteria at the time of enrollment, their participation in the CAPRISA 004 trial was continued subject to their termination in the other trials.
Following the unblinding of the treatment allocation on study completion in June 2010, it was established that of the 185 ineligible participants, 94 (50.8\%) were assigned to the tenofovir arm and 91 (49.2\%) to the placebo arm. Of the 889 eligible participants included in the primary intent to treat analysis for CAPRISA 004 [9], ineligible participants were older, had a lower monthly income, had a greater mean parity, reported having more sex and more condom use with sex and had fewer adverse events reported (all $\mathrm{p}<0.001$ ) compared to eligible participants (Table 1 ). We found no differences in the primary outcome of HIV infection or secondary outcome of pregnancy rates (Table 1 ).

\subsection{Communication with key stake holders}

A full audit was completed within three weeks of the coenrollment discovery and several consultations were held between CAPRISA and neighbouring AIDS research unit. The CAPRISA 004 Data Safety and Monitoring Board (DSMB) and regulatory authorities such as the University of KwaZuluNatal Biomedical Research Ethics Committee (BREC), Family Health International Protection of Human Subjects Committee (FHI-PHSC), and the South African Medicines Control Council (MCC) were notified. In addition, other groups conducting research within a $100 \mathrm{~km}$ radius, local community groups, advocacy organizations, the CAPRISA Research Support Groups and CAPRISA's community liaison team were also notified. Early in May 2008 the Microbicides Trial Network released a statement on the ineligible participants enrolled in the CAPRISA 004 trial [17].

Based on the International Conference on Harmonization (ICH) guidelines E9 on inclusion and exclusion criteria [18] and in consultation with the study statisticians and the DSMB the Principal investigators took the decision, to withdraw all participants identified as ineligible and enrolled in the CAPRISA 004 trial. Study staff, site staff and all trial participants were informed on the decision and all ineligible enrolled participants were terminated from CAPRISA 004.

The DSMB additionally recommended that the trial continue and advised the study team to:

- Retain women who enrolled in another study after enrolling in the CAPRISA 004 trial $(n=4)$.

- Take appropriate measures to achieve study objectives in the shortest amount of time by increasing the sample size to replace participants who had been terminated.

- Observe standard exit procedures for ineligible enrolled participants being withdrawn, including safety assessment.

- Report to the DSMB on co-enrollments at subsequent meetings, including whether or not there were more attempts at co-enrollment and/or the number of $\mathrm{Co}^{-}$ enrollments averted.

\section{Lessons learnt}

\subsection{Changes in study procedures}

A number of innovative measures were developed to remove the possibility of further co-enrollments. Firstly, study staff completed training to understand the disadvantages of co-enrollments and screening for co-enrollments 
Table 1

Comparison of characteristics of CAPRISA 004 ineligible (co-enrolled and not co-enrolled) and eligible trial participants $(\mathrm{N}=1074)$.

\begin{tabular}{|c|c|c|c|}
\hline Characteristics & $\begin{array}{l}\text { Ineligible co-enrolled and } \\
\text { not co-enrolled }{ }^{\mathrm{a}} \mathrm{n}=185\end{array}$ & $\begin{array}{l}\text { Eligible enrolled }^{\mathrm{b}} \\
\mathrm{n}=889\end{array}$ & p-Value \\
\hline \multicolumn{4}{|l|}{ Baseline sociodemographic } \\
\hline Mean age & 25.6 years & 23.9 years & $<0.001$ \\
\hline High school education completed or more & $88(47 \%)$ & $365(41 \%)$ & 0.120 \\
\hline Monthly income $<$ R1000 & $140(75.7 \%)$ & $718(80.8 \%)$ & 0.001 \\
\hline Married & $13(7.0 \%)$ & $50(5.6 \%)$ & 0.491 \\
\hline Stable partner & $153(82.7 \%)$ & $783(88.1 \%)$ & 0.053 \\
\hline Mean parity & 1.5 & 1.1 & $<0.001$ \\
\hline \multicolumn{4}{|l|}{ Baseline sexual behaviour } \\
\hline Mean age sexual debut & 17.7 years & 17.4 years & 0.093 \\
\hline Mean number of lifetime sexual partners & 3.8 & 3.3 & 0.548 \\
\hline Mean age of oldest partner in the last 30 days & 30.0 years & 27.4 years & $<0.001$ \\
\hline Reported sex in the past 7 days & $155(83.8 \%)$ & $550(61.9 \%)$ & $<0.001$ \\
\hline Always use condom during sex & $84(45.4 \%)$ & $259(29.1 \%)$ & $<0.001$ \\
\hline Anal sex in the past 30 days & $0(0.0 \%)$ & $4(0.4 \%)$ & 1.000 \\
\hline New sex partners in the past 30 days & $5(2.7 \%)$ & $10(1.1 \%)$ & 0.160 \\
\hline Sex partners had other sex partners in the past 30 days & $33(17.8 \%)$ & $179(20.1 \%)$ & 0.591 \\
\hline More than 2 casual sex partners in the past 30 days & $5(2.7 \%)$ & $20(2.2 \%)$ & 0.788 \\
\hline Ever forced to have sex with anyone & $2(1.1 \%)$ & $6(0.7 \%)$ & 0.632 \\
\hline Ever received money in exchange for sex & $5(2.7 \%)$ & $17(1.9 \%)$ & 0.565 \\
\hline Knows sex partner had an HIV test in the last 30 days & $4(2.2 \%)$ & $19(2.1 \%)$ & 0.188 \\
\hline Alcohol consumed before last sex & $6(3.2 \%)$ & $38(4.3 \%)$ & 0.684 \\
\hline Genital signs and symptoms in the past 30 days & $65(35.1 \%)$ & $335(37.7 \%)$ & 0.559 \\
\hline \multicolumn{4}{|l|}{ Study arm allocation } \\
\hline Tenofovir gel & $94(50.8 \%)$ & $445(50.1 \%)$ & 0.872 \\
\hline Placebo & $91(49.2 \%)$ & 444 (49.9\%) & \\
\hline \multicolumn{4}{|l|}{ Primary endpoint } \\
\hline HIV incidence/100 women years (95\% CI ) & $6.5(2.1-15.1)$ & $7.3(5.9-8.9)$ & 0.637 \\
\hline \multicolumn{4}{|l|}{ Secondary endpoints } \\
\hline Adverse events/participant & $155 / 89(48.1 \%)$ & $4692 / 838(94.3 \%)$ & $<0.001$ \\
\hline Serious adverse events/participant & $3 / 3(1.6 \%)$ & $39 / 37(4.2 \%)$ & 0.132 \\
\hline Pregnancy incidence/100 women years (95\% CI) & $5.1(1.4-13.2)$ & $4.0(3.0-5.2)$ & 0.786 \\
\hline
\end{tabular}

a Ineligible co-enrolled: participants who were participating in two HIV prevention trials simultaneously. Ineligible not co-enrolled: participants who had participated in a trial of a vaginally applied product within the last 12 months.

b Eligible enrolled: participants who met all the eligibility criteria for enrollment into CAPRISA 004 trial.

and to be vigilant during participant study visits. Participant scripts were created to ensure that the concept of coenrollment was reinforced during all information and informed consent sessions and at all study visits. It was critically important that participants understood the consequences of co-enrollments especially in trials of investigational products to potentially endanger their safety, confound safety monitoring of individual participants and compromise the scientific integrity of all studies that the participant enrolled in. Furthermore, additional counselling was instituted to ensure that eligible enrolled participants understood the risks of co-enrolling and did not enroll in other trials during the course of their participation in CAPRISA 004 trial.

Study related information material used at screening, enrollment informed consent forms were modified to provide explicit information of the dangers of co-enrollment. Participants signed a declaration in the informed consent confirming that they were not enrolled in another study of an investigational drug. We administered a structured questionnaire to assess co-enrollment in all participants. All revised documents and informed consents were approved by BREC prior to administration.

Importantly, the neighbouring AIDS research unit generously modified and shared its existing participant database with CAPRISA for confirming the unique South African identification numbers (SA ID) using a secured password protected and restricted user access electronic database. The use of this shared database for checking of SA IDs of CAPRISA 004 volunteers to verify if they were currently participating in any other trial that was testing an investigational product was approved by UKZN BREC. No other study related personal or other information was shared between studies and organizations. The database was accessed through a common server and included a complete listing of HIV prevention studies being conducted in this region. The database included sections specifying date of enrollment and exit date from the specific study to determine if the potential participant has been enrolled in any of the existing studies in this shared database. If a volunteer was identified as already participating in a study of an investigational product she would be informed accordingly and all further pre-enrollment procedures were halted and the volunteer classified as ineligible based on the exclusionary criteria.

The CAPRISA pharmacy maintains an audit trail in gel dispensing, verification of gel variant allocation and the return of used and unused applicators at each monthly visit. Whilst these measures had been set up to ensure the availability of good quality product at all times and to assist with enhancing measures of adherence, the pharmacy procedures also identified a gel applicator from another trial returned erroneously as a CAPRISA 004 applicator. The high 
level of vigilance from pharmacy staff was an important ongoing adjunct to the linked database.

\subsection{Greater awareness of recruitment populations}

Whilst the priority population for the urban CAPRISA Clinic site was the STD clinic, community recruitment and community volunteers were allowed. As the CAPRISA 004 protocol allowed for transitioning of participants from other CAPRISA cohort studies with no investigational product, CAPRISA 004 recruitment staff incorrectly assumed that prior participation in trials of investigational drugs or behaviour modification for HIV prevention was also acceptable. Additionally the screening process did not provide sufficient information on the risks associated with simultaneous use of multiple investigational products through participation in multiple trials.

\subsection{Participant-related factors that contributed to co- enrollment}

Whilst every effort was made to ensure that volunteers understood trial-related procedures and the implication of trial participation, understanding why people join trials is important. Following individual interviews $(\mathrm{n}=13)$ and focus group discussions $(n=2)$ with site staff and exit interviews conducted with co-enrolled participants, key themes emerged as contributory to participant's seeking enrollment in multiple trials.

\subsubsection{High-quality health care as an incentive}

Whilst public sector primary health care services provide free contraceptives, HIV testing services and treatment for sexually transmitted infections at no cost, under-developed health facilities, overworked staff and drug shortages are a reality that impact access to and quality of health services for indigent populations. The high quality care with emphasis on attention to detail in the trial was an important benefit women were unwilling to lose once their follow-up in a particular trial ended.

\subsubsection{Financial incentive}

Some of the research participants were unemployed or if employed, had low earning capacity. For some of the women who had little or no income, the amount of R150 per study visit that was specified by the government regulatory body, the Medicines Control Council, was sufficient incentive for study participation $[19,20]$. As multiple trial sites arise within defined geographical areas, a network of astute research participants can keep each other informed of clinical trial opportunities, with some participants even acting as selfappointed recruiters providing information on where clinical trials are actively enrolling. Women co-enrolling in multiple trials can skillfully respond to questions to avoid being identified as co-enrollees, and some have even worked out ways of reducing study visit time.

\subsubsection{Altruism}

Many participants came from communities being devastated by the HIV/AIDS epidemic, and some felt a strong imperative to contribute to finding solutions to the epidemic.
The women were able to articulate their altruistic motivation for trial participation and their tangible need to contribute to the urgent need to find options for women. For them clinical trial participation provided an opportunity to contribute to finding options to prevent HIV infection in women, and they felt that they were making a contribution through their participation.

\subsubsection{Seeking gel for a better sex life}

Some women mentioned that their sexual experiences had improved since initiating gel use. Some women, who knew that the trial they were in would be ending soon. learnt about the CAPRISA 004 trial and joined it to be able to continue with gel use. Some women said that they wanted to co-enroll because they felt that this could increase their chances of getting an active product instead of the placebo.

\subsubsection{Low risk of discovery and peer pressure}

Some women knew that others had successfully coenrolled without being identified. Furthermore, these women were aware that trial staff had no way of knowing whether they used the study product or not, and elected not to use any trial study product. They rationalized that they were not increasing their personal risk in any way because they were not using any product, were attending study visits and undergoing study procedures.

Many women understood that their participation in the other trials they were already enrolled in was coming to an end and that overlaps between the two trials were minimal and worth the risk and effort to secure high quality care, income or access to study gel that they had become accustomed to. Some mentioned peer-pressure from fellow co-enrollees as a reason for their co-enrollment. Importantly, most co-enrolled participants knew that they were doing something that was not allowed.

\section{Conclusions}

Participation in HIV prevention trials is often time consuming and involves commitment by participants to make multiple visits, endure clinical procedures and adhere to the investigational product regimen. However, women are financially reimbursed and provided with quality care at the trial sites such as access to family planning, treatment of sexually transmitted infections, access to caring clinicians, and appropriate counselling and referrals. Significantly the majority of study participants were honest in their self-report on non-enrollment in other studies of investigational drugs.

Co-enrollment has important implications for both the trial participants and the integrity of the trial. Clinically, exposure to more than one investigational product poses safety concerns for the participants, and it becomes impossible to attribute adverse events to one or another trial product. Scientifically, co-enrollment may attenuate or synergize the impact of a trial product and invalidate the measure of effect. Although participants are made aware of the potential dangers of co-enrollment during the informed consent process, this was insufficient to prevent co-enrollment in the CAPRISA 004 trial.

Women attending a study clinic have many opportunities to share information with each other and proximate clinical 
trial sites incubate such networking. Women who co-enrolled generally did so intentionally and for a number of very rational reasons. The study team had to react quickly and decisively to disqualify co-enrollees and preclude future ineligible enrollments in the CAPRISA 004 trial.

The likelihood of co-enrollment was not fully appreciated by study staff prior to inception, and systems for identifying and preventing ineligible enrollment were poorly developed. The importance of a database system shared amongst nearby research organizations is now apparent, and is a prominent part of planning any future trials in Durban.

As South Africa will continue to host large-scale HIV prevention trials, collegial inter-organizational collaboration will be necessary to eliminate co-enrollment. Shared registries are essential to protect the safety of trial participants and validity of these vital studies.

\section{Acknowledgements}

The CAPRISA 004 Tenofovir gel trial was supported by the Centre for the AIDS Programme of Research in South Africa (CAPRISA), the United States Agency for International Development (USAID), FHI (cooperative agreement \# GPOA-00-05-00022-00, contract \# 132119), and LIFElab, a biotechnology division of the South African Department of Science and Technology. Support from CONRAD for the product manufacturing and packaging as well as support from Gilead Sciences for the Tenofovir used in the production of gel is gratefully acknowledged. We thank the US National Institutes for Health's Comprehensive International Program of Research on AIDS (CIPRA grant \# AI51794) and the Columbia University-Southern African Fogarty AIDS International Training and Research Programme (AITRP grant \# D43TW00231) for the research infrastructure and training that made this trial possible.

We sincerely thank the women who participated in the CAPRISA 004 Tenofovir gel trial for their dedication and commitment. Special thanks to the CAPRISA eThekwini clinic site staff and to members of the Community Research Support Groups. A special thanks to Dr Paul Feldblum of FHI for reviewing the manuscript and his valuable editorial comments.

\section{References}

[1] UNAIDS World Health Organization. AIDS epidemic update: November 2009. "UNAIDS/0936E/JC1700E" ISBN 9789291738328 (NLM classification: WC 50341); 2009.

[2] Padian NS, Buve A, Balkus J, Serwadda D, Cates Jr W. Biomedical interventions to prevent HIV infection: evidence, challenges, and way forward. Lancet 2008;372:585-99.
[3] Padian NS, McCoy SI, Balkus JE, Wasserheit JN. Weighing the gold in the gold standard: challenges in HIV prevention research. AIDS 2010;24: 621-35.

[4] Evans D. Hierarchy of evidence: a framework for ranking evidence evaluating healthcare interventions. Journal of Clinical Nursing 2003;12:77-84.

[5] Skoler-Karpoff S, Ramjee G, Ahmed K, Altini L, Plagianos MG, Friedland $B$, et al. Efficacy of Carraguard for prevention of HIV infection in women in South Africa: a randomised, double-blind, placebo-controlled trial. Lancet 2008;372:1977-87.

[6] Van Damme L, Govinden R, Mirembe FM, Guedou F, Solomon S, Becker ML, et al. Lack of effectiveness of cellulose sulfate gel for the prevention of vaginal HIV transmission. The New England Journal of Medicine 2008;359:463-72.

[7] Van Damme L, Ramjee G, Alary M, Vuylsteke B, Chandeying V, Rees H, et al. Effectiveness of COL-1492, a nonoxynol-9 vaginal gel, on HIV-1 transmission in female sex workers: a randomised controlled trial. Lancet 2002;360:971-7.

[8] Padian NS, van der Straten A, Ramjee G, Chipato T, de Bruyn G, Blanchard K, et al. Diaphragm and lubricant gel for prevention of HIV acquisition in southern African women: a randomised controlled trial. Lancet 2007:370:251-61.

[9] Abdool Karim Q Abdool Karim SS, Frohlich JA, Grobler AC, Baxter C, Mansoor LE, et al. Effectiveness and safety of tenofovir gel, an antiretroviral microbicide, for the prevention of HIV infection in women. Science 2010;329:1168-74 New York, NY.

[10] Abdool Karim SS, Coletti A, Richardson B, Ramjee G, Hoffman I, Chirenje $\mathrm{M}$, et al. Safety and Effectiveness of Vaginal Microbicides BufferGel and PRO $2000 \mathrm{Gel}$ for the Prevention of HIV Infection in Women: Results of the HPTN 035 Trial. [abstract 48lb]. Conference on Opportunistic Infections and Retroviruses. 8 February 2009; Montreal; 2009.

[11] Almeida L, Azevedo B, Nunes T, Vaz-da-Silva M, Soares-da-Silva P. Why healthy subjects volunteer for phase I studies and how they perceive their participation? European Journal of Clinical Pharmacology 2007;63:1085-94.

[12] McCann SK, Campbell MK, Entwistle VA. Reasons for participating in randomised controlled trials: conditional altruism and considerations for self. Trials 2010;11:31.

[13] Colfax G, Buchbinder S, Vamshidar G, Celum C, McKirnan D, Neidig J et al. Motivations for participating in an HIV vaccine efficacy trial. Journal of Acquired Immune Deficiency Syndromes 2005;39:359-64.

[14] Boyar D, Goldfarb N. Preventing overlapping enrollment in clinical studies. Journal of Clinical Research Best Practices 2010;6:1-4.

[15] Abdool Karim Q, Kharsany A, Frohlich J, Werner L, Mashego M, Mlotshwa M, et al. Stabilizing HIV prevalence masks high HIV incidence rates amongst rural and urban women in KwaZulu-Natal, South Africa. International Journal of Epidemiology, online November 3, 2010, doi: 10.1093/ije/dyq176.

[16] van Loggerenberg F, Mlisana K, Williamson C, Auld SC, Morris L, Gray $\mathrm{CM}$, et al. Establishing a cohort at high risk of HIV infection in South Africa: challenges and experiences of the CAPRISA 002 acute infection study. PLoS ONE 2008;3:e1954.

[17] Sharon Hillier PI. Statement on co-enrollment of participants in CAPRISA 004 and HPTN 035 in South Africa. (Microbicide Trials Network); May 52008.

[18] International Conference on Harmonisation Guidelines E9. International conference on harmonisation of technical requirements for registration of pharmaceuticals for human use. ICH harmonised tripartate guideline statistical principles for clinical trials-E9; Feb. 51998.

[19] Burgess L Sulzer N, Emanuel S. Clinical trial remuneration: the patients' perspective. South African Medical Journal 2008;98:95-7.

[20] Koen J, Slack C, Barsdorf N, Essack Z. Payment of trial participants can be ethically sound: moving past a flat rate. South African Medical Journal 2008;98:926-9. 